\title{
A maximally superintegrable system on an $n$-dimensional space of nonconstant curvature
}

\author{
Á. Ballesteros ${ }^{a *}$ \\ A. $\operatorname{Enciso}^{b \dagger}$ \\ F. J. Herranz ${ }^{a \ddagger}$ \\ O. Ragnisco ${ }^{c \S}$ \\ ${ }^{a}$ Depto. de Física, Universidad de Burgos, 09001 Burgos, Spain \\ ${ }^{b}$ Depto. de Física Teórica II, Universidad Complutense, 28040 Madrid, Spain \\ ${ }^{c}$ Dip. di Fisica, Università di Roma 3, and Instituto Nazionale di Fisica Nucleare \\ Via Vasca Navale 84, 00146 Rome, Italy
}

\begin{abstract}
A novel Hamiltonian system in $n$ dimensions which admits the maximal number $2 n-1$ of functionally independent, quadratic first integrals is presented. This system turns out to be the first example of a maximally superintegrable Hamiltonian on an $n$-dimensional Riemannian space of nonconstant curvature, and it can be interpreted as the intrinsic Smorodinsky-Winternitz system on such a space. Moreover, we provide three different complete sets of integrals in involution and solve the equations of motion in closed form.
\end{abstract}

\section{Introduction}

A Hamiltonian system on a $2 n$-dimensional phase space is maximally superintegrable (MS) if it is Liouville integrable [2] and there exist further $n-1$ functionally independent (global) first integrals. Throughout this paper we shall restrict ourselves to the case of natural Hamiltonians, whose kinetic term defines a Riemannian metric on an $n$-dimensional configuration space.

The intrinsic relevance of MS systems has resulted in a wealth of results concerning MS Hamiltonians [11,20,21,28,34]. MS models with integrals of motion quadratic in the momenta are of particular interest because of their connections with generalized symmetries $[19,35]$, isochronic potentials [10,18], and separability of the associated Hamilton-Jacobi (HJ) equation and Schrödinger equations $[8,22,27]$. A systematic study of MS systems on certain low-dimensional spaces is currently being developed by Kalnins et al. (cf. [24] and references therein), and in fact in two dimensions they managed to obtain a (local) classification of all MS Hamiltonians with integrals at most quadratic in the momenta $[25,26]$. This result, which hinges on a classical theorem by Koenigs [29],

\footnotetext{
*angelb@ubu.es

†aenciso@fis.ucm.es

‡fjherranz@ubu.es

§ragnisco@fis.uniroma3.it
} 
yielded the first examples of MS systems not defined on constant curvature spaces.

Unfortunately, most examples of MS Hamiltonians are restricted to lowdimensional spaces. To the best of our knowledge, the only known examples of $n$ dimensional MS systems with quadratic integrals are given by the (generalized) Kepler problem [14], the Smorodinsky-Winternitz (SW) system [15,17], their generalizations to the simply connected spaces of constant sectional curvature (cf. [4] and references therein), and the geodesic flow on these spaces. Note that the allowed potentials correspond essentially to those appearing in the extension to space forms of Bertrand's theorem [7,31]. If one drops the requirement that the integrals be quadratic in momenta, the list can be enlarged with the rational and hyperbolic Calogero-Sutherland-Moser models of type $A[9,32,36$, 38 ], the nonisotropic oscillator with rational frequencies and the nonperiodic Toda lattice $[1,37]$.

In this paper we show that the Hamiltonian

$$
\mathcal{H}(\mathbf{p}, \mathbf{q})=\frac{\mathbf{p}^{2}+\omega^{2} \mathbf{q}^{2}+\sum_{j} b_{j} q_{j}^{-2}}{\kappa+\mathbf{q}^{2}},
$$

where $\kappa>0$ and $\omega^{2}, b_{j} \geq 0$, is a MS system with quadratic first integrals. Here $\mathbf{p}, \mathbf{q} \in \mathbb{R}^{n}$, and we shall always assume that $\mathbb{R}^{2 n}$ is endowed with its standard symplectic form $\mathrm{d} \mathbf{q} \wedge \mathrm{d} \mathbf{p}$. For any $\mathbf{u} \in \mathbb{R}^{n}$ we use the notation

$$
\mathbf{u}^{2}=\mathbf{u} \cdot \mathbf{u}=\sum_{i} u_{i}^{2}
$$

and all the sum indices run from 1 to $n$ unless otherwise stated.

The Hamiltonian (1) yields the motion under the potential

$$
V(\mathbf{q})=\frac{\omega^{2} \mathbf{q}^{2}+\sum_{j} b_{j} q_{j}^{-2}}{\kappa+\mathbf{q}^{2}}
$$

on the conformally flat Riemannian manifold $\mathcal{M}^{n}:=\left(\mathbb{R}^{n}, \mathrm{~d} s^{2}\right)$ whose metric is given by

$$
\mathrm{d} s^{2}=\left(\kappa+\mathbf{q}^{2}\right) \mathrm{d} \mathbf{q}^{2} .
$$

The Hamiltonian (11) provides the first example of a Hamiltonian system on a Riemannian space of nonconstant curvature which is MS in any dimension. The analysis of the geometry of $\mathcal{M}^{n}$ presented in Sec. 4 shows that this model can be in fact regarded as the intrinsic SW system on this space. When $n=2$ this model is listed in Kalnins et al.'s classification of MS systems in the Darboux space of type III [26]. This model is singled out among the others in this list because it is the only one readily amenable to the Poisson coalgebra treatment developed in Sec. 2

The paper is organized as follows. In Sec. 2 we show that the Hamiltonian (1) is indeed MS and briefly study the separability of the HJ equation. The proof is greatly simplified by the use of the $\mathfrak{s l}(2)$ Poisson coalgebra symmetry $[5,6]$ of the model. In Sec. 3 we study the equations of motion and find their general solution in closed form using the previously computed first integrals. Quite remarkably, the radial motion is essentially that of the Kepler problem, whereas the HJ equation essentially coincides with the one for the SW system. In Sec. प we analyze the geometry of the underlying Riemannian space and relate the Hamiltonian (1) to the intrinsic harmonic oscillator in $\mathcal{M}^{n}$. 


\section{Maximal superintegrability and separability of the HJ equation}

Instead of dealing with the model (1) directly, in the following two sections we prefer to analyze the Hamiltonian

$$
H(\mathbf{p}, \mathbf{q})=\frac{\mathbf{p}^{2}-c+\sum_{j} b_{j} q_{j}^{-2}}{2\left(\kappa+\mathbf{q}^{2}\right)}, \quad c:=\kappa \omega^{2},
$$

which is related to (1) via $\mathcal{H}=2 H+\omega^{2}$. Obviously the maximal superintegrability of $H$ is equivalent to that of $\mathcal{H}$, whereas the trajectories of $H$ can be mapped into those of $\mathcal{H}$ via the time dilation $t \mapsto t / 2$. In particular, $H$ and $\mathcal{H}$ possess the same orbits.

A first observation is that there is an $\mathfrak{s l}(2)$ Poisson coalgebra symmetry of the Hamiltonian (4) that immediately provides $2 n-2$ quadratic integrals. This underlying coalgebra structure is shared by all the other known examples of quadratically MS models [3,4], as well as by uncountably many other integrable Hamiltonian systems which are not MS. Therefore, the crucial result in this section is the derivation of an additional first integral, functionally independent of the previous $2 n-2$.

The $\mathfrak{s l}(2)$ Poisson coalgebra is defined by the basis $\left\{J_{\epsilon}: \epsilon= \pm, 0\right\}$ together with the following Lie-Poisson brackets, Casimir $C$ and primitive coproduct $\Delta$ :

$$
\begin{gathered}
\left\{J_{0}, J_{+}\right\}=2 J_{+}, \quad\left\{J_{0}, J_{-}\right\}=-2 J_{-}, \quad\left\{J_{-}, J_{+}\right\}=4 J_{0}, \\
C=J_{-} J_{+}-J_{0}^{2}, \quad \Delta\left(J_{\epsilon}\right)=J_{\epsilon} \otimes 1+1 \otimes J_{\epsilon} .
\end{gathered}
$$

A symplectic (one-dimensional) realization of the algebra (5) on $\left(\mathbb{R}^{2}, \mathrm{~d} q_{1} \wedge \mathrm{d} p_{1}\right)$ is given by

$$
J_{-}=q_{1}^{2}, \quad J_{0}=q_{1} p_{1}, \quad J_{+}=p_{1}^{2}+b_{1} q_{1}^{-2},
$$

where $b_{1}$ is a real parameter labeling the representation which corresponds to the value of the (one-dimensional) Casimir. Under this realization, the Lie-Poisson product of the $\mathfrak{s l}(2)$ algebra is recovered by computing the corresponding Poisson bracket in $\left(\mathbb{R}^{2}, \mathrm{~d} q_{1} \wedge \mathrm{d} p_{1}\right)$. Moreover, its $n$-th coproduct yields a symplectic realization in $\mathbb{R}^{2 n}$ via

$$
J_{-}=\mathbf{q}^{2}, \quad J_{0}=\mathbf{p} \cdot \mathbf{q}, \quad J_{+}=\mathbf{p}^{2}+\sum_{j} b_{j} q_{j}^{-2},
$$

with $b_{j} \in \mathbb{R}$. Again, the Lie-Poisson product is given by the standard Poisson bracket

$$
\{f, g\}=\frac{\partial f}{\partial \mathbf{q}} \frac{\partial g}{\partial \mathbf{p}}-\frac{\partial f}{\partial \mathbf{p}} \frac{\partial g}{\partial \mathbf{q}}
$$

and the Casimir (6) is simply

$$
C=\mathbf{L}^{2}+\sum_{j} \frac{b_{j} \mathbf{q}^{2}}{q_{j}^{2}}
$$

where $\mathbf{L}^{2}$ denotes the angular momentum. From this expression it is apparent that $C$ is a homogenous function of degree 0 . 
This $\mathfrak{s l}(2) \otimes \cdots \otimes \mathfrak{s l}(2)$ symmetry endows any $n$-dimensional Hamiltonian in the enveloping algebra with $2 n-3$ integrals other than the Hamiltonian given by the left and right partial Casimirs. More precisely we have the following result, which we quote from Ref. [3].

Theorem 1. Any Hamiltonian $H_{0}(\mathbf{p}, \mathbf{q})=h\left(J_{+}, J_{0}, J_{-}\right)$possesses $2 n-3$ first integrals given by the left and right partial Casimirs

$$
\begin{aligned}
C^{(m)} & =\sum_{1 \leq i<j \leq m}\left[\left(q_{i} p_{j}-q_{j} p_{i}\right)^{2}+\frac{b_{i} q_{j}^{2}}{q_{i}^{2}}+\frac{b_{j} q_{i}^{2}}{q_{j}^{2}}\right]+\sum_{i=1}^{m} b_{i} \\
C_{(m)} & =\sum_{n-m<i<j \leq n}\left[\left(q_{i} p_{j}-q_{j} p_{i}\right)^{2}+\frac{b_{i} q_{j}^{2}}{q_{i}^{2}}+\frac{b_{j} q_{i}^{2}}{q_{j}^{2}}\right]+\sum_{i=n-m+1}^{n} b_{i},
\end{aligned}
$$

where $1<m \leq n$. Here $C^{(n)}=C_{(n)}=C$ is the Casimir (6) and the functions

$$
\left\{H_{0}, C^{(l)}, C_{(m)}: 1<l<n, 1<m \leq n\right\}
$$

are functionally independent. Moreover, the subsets $\left\{H_{0}, C^{(m)}: 1<m \leq n\right\}$ and $\left\{H_{0}, C_{(m)}: 1<m \leq n\right\}$ are in involution.

The previous result obviously applies to the Hamiltonian (4), which corresponds to

$$
H=\frac{J_{+}-c}{2\left(\kappa+J_{-}\right)} .
$$

The main result of this paper is that the remaining first integral, which makes $H$ MS, can indeed be found easily.

Theorem 2. The remaining first integral can be chosen as

$$
I_{i}(\mathbf{p}, \mathbf{q})=p_{i}^{2}-2 H(\mathbf{p}, \mathbf{q}) q_{i}^{2}+b_{i} q_{i}^{-2},
$$

for any $1 \leq i \leq n$. Moreover, the set $\left\{I_{i}: 1 \leq i \leq n\right\}$ is also in involution.

Proof. The equations of motion under $H$ are given by

$$
\begin{aligned}
\dot{q}_{i} & =\frac{p_{i}}{\kappa+\mathbf{q}^{2}}, \\
\dot{p}_{i} & =\frac{2 H q_{i}+b_{i} q_{i}^{-3}}{\kappa+\mathbf{q}^{2}} .
\end{aligned}
$$

Combining both equations one can write

$$
\left(\kappa+\mathbf{q}^{2}\right) \ddot{q}_{i}+2(\mathbf{q} \cdot \dot{\mathbf{q}}) \dot{q}_{i}-\frac{2 H q_{i}+b_{i} q_{i}^{-3}}{\left(\kappa+\mathbf{q}^{2}\right)}=0 .
$$

Multiplying this equation by $2\left(\kappa+\mathbf{q}^{2}\right) \dot{q}_{i}$ one immediately finds

$$
\frac{\mathrm{d}}{\mathrm{d} t}\left[\left(\kappa+\mathbf{q}^{2}\right)^{2} \dot{q}_{i}^{2}-2 H q_{i}^{2}+b_{i} q_{i}^{-2}\right]=0,
$$


which yields the desired result. The functional independence of $I_{i}$ is easily established through a tedious but straightforward computation. The fact that $\left\{I_{i}\right\}$ are in involution follows from

$$
\begin{aligned}
\left\{I_{i}, I_{j}\right\}=-2 q_{i}^{2}\left(\left\{H, p_{j}^{2}\right\}-2 H\left\{H, q_{j}^{2}\right\}+b_{j}\left\{H, q_{j}^{-2}\right\}\right) & \\
& +2 q_{j}^{2}\left(\left\{H, p_{i}^{2}\right\}-2 H\left\{H, q_{i}^{2}\right\}+b_{i}\left\{H, q_{i}^{-2}\right\}\right) \\
= & -2 q_{i}^{2}\left\{H, I_{j}\right\}+2 q_{j}^{2}\left\{H, I_{i}\right\}=0 .
\end{aligned}
$$

Quadratic integrability is linked to the separability of the HJ equation by a theorem due to Kalnins and Miller [27]. In fact, it is not difficult to show that this equation is actually superseparable. If we write $S(t, \mathbf{q})=W(\mathbf{q})-\frac{1}{2} E t$ the HJ equation for $H$ reads

$$
\left(\frac{\partial W}{\partial \mathbf{q}}\right)^{2}-E \mathbf{q}^{2}+\sum_{j} b_{j} q_{j}^{-2}=c+\kappa E .
$$

Thus one essentially recovers the HJ equation for the SW system with a different set of constants, namely

$$
H_{\mathrm{SW}}(\mathbf{p}, \mathbf{q})=\mathbf{p}^{2}-E \mathbf{q}^{2}+\sum_{j} b_{j} q_{j}^{-2} .
$$

Therefore,

Proposition 3. $H$ separates in the same coordinate systems as the $S W$ system.

In three dimensions, e.g., it separates in 8 out of the 11 possible orthogonal coordinate systems $[16,23]$. As a matter of fact, it has long been conjectured that any MS system should separate in multiple coordinate systems, but to our best knowledge this general claim has not been proved or disproved.

\section{Integration of the equations of motion}

In this section we shall compute the trajectories of the Hamiltonian system (4) in closed form. It is convenient to start by exploiting the $\mathfrak{s l}(2)$ symmetry to compute the evolution of the radial variable

$$
x=\kappa+J_{-}=\kappa+\mathbf{q}^{2} .
$$

We use the notation

$$
E:=2 H=\frac{J_{+}-c}{x}
$$

and assume $c \neq 0$.

The evolution of the $\mathfrak{s l}(2)$ generators (7) is given by the following set of equations:

$$
\begin{aligned}
\dot{x} & =\left\{J_{-}, H\right\}=\frac{2 J_{0}}{x}, \\
\dot{J}_{0} & =\left\{J_{0}, H\right\}=\frac{J_{+}+E(x-\kappa)}{x}, \\
\dot{J}_{+} & =\left\{J_{+}, H\right\}=\frac{2 E J_{0}}{x} .
\end{aligned}
$$


Using these equations one can write the Casimir (6) as

$$
C=-\frac{1}{4} x^{2} \dot{x}^{2}+E x^{2}+(c-E \kappa) x-c \kappa \geq 0 .
$$

For the sake of concreteness, let us assume that $E>0$ and set

$$
\begin{aligned}
\alpha & :=\frac{1}{2}\left(\kappa-\frac{c}{E}\right), \\
\gamma^{2} & :=\frac{1}{4}\left(\kappa+\frac{c}{E}\right)^{2}+\frac{C}{E} .
\end{aligned}
$$

In this case

$$
x^{2} \dot{x}^{2}=4 E\left[(x-\alpha)^{2}-\gamma^{2}\right],
$$

and one can easily integrate this equation as

$$
\pm 2 \sqrt{E}(t-\tau)=\sqrt{(x-\alpha)^{2}-\gamma^{2}}+\alpha \cosh ^{-1}\left(\frac{x-\alpha}{\gamma}\right) .
$$

Here $\tau$ is an arbitrary constant and $\gamma$ is the positive square root of $\gamma^{2}$. Note that the equation (14) for the variable $x$ (the squared radius) coincides with the radial equation in the Kepler problem.

The above expression yields $t$ as a monotonic function of $x$, so this relation is globally invertible (in each half-orbit). However, it is not possible to obtain the inverse function $x(t)$ in closed form. Hence we prefer to parametrize the trajectory by the radial variable $x$. In terms of this variable, the first integral (8) reads

$$
I_{i}=4 E\left[(x-\alpha)^{2}-\gamma^{2}\right]\left(\frac{\mathrm{d} q_{i}}{\mathrm{~d} x}\right)^{2}-E q_{i}^{2}+b_{i} q_{i}^{-2} .
$$

Let us set

$$
Q_{i}=q_{i}^{2}, \quad \alpha_{i}=-\frac{I_{i}}{2 E}, \quad \gamma_{i}^{2}=\alpha_{i}^{2}+E^{-1} b_{i}
$$

so that

$$
E q_{i}^{4}+I_{i} q_{i}^{2}-b_{i}=E\left[\left(Q_{i}-\alpha_{i}\right)^{2}-\gamma_{i}^{2}\right] .
$$

Then one can perform the integration of Eq. (16) to obtain

$$
\int \frac{\mathrm{d} Q_{i}}{\sqrt{\left(Q_{i}-\alpha_{i}\right)^{2}-\gamma_{i}^{2}}}=\varphi_{i}+\int \frac{\mathrm{d} x}{\sqrt{(x-\alpha)^{2}-\gamma^{2}}},
$$

i.e.,

$$
\cosh ^{-1}\left(\frac{Q_{i}-\alpha_{i}}{\gamma_{i}}\right)=\varphi_{i}+\cosh ^{-1}\left(\frac{x-\alpha}{\gamma}\right)
$$

where $\varphi_{i}$ are constants and $\gamma_{i} \geq 0$. The full solution of this equation is therefore given by

$$
\begin{aligned}
Q_{i} & =\alpha_{i}+\gamma_{i} \cosh \left(X+\varphi_{i}\right) \\
& =\alpha_{i}+\gamma^{-1} \gamma_{i} \cosh \varphi_{i}(x-\alpha)+\gamma_{i} \sinh \varphi_{i}\left|1-\left(\frac{x-\alpha}{\gamma}\right)^{2}\right|^{1 / 2},
\end{aligned}
$$

with $X:=\cosh ^{-1}\left(\gamma^{-1}(x-\alpha)\right)$. Eqs. (15) and (18) are the main results of this section. 
All the orbits with positive energy are recovered through an appropriate choice of the $2 n$ parameters

$$
\left\{\tau, \alpha_{i}, \varphi_{j}: 1 \leq i \leq n-1,1 \leq j \leq n\right\},
$$

where $\tau$ was defined in Eq. (15). In what follows we shall express the remaining quantities in terms of this fundamental set.

If we sum over $i$ in Eq. (18) and use that $x=\kappa+\sum_{i} Q_{i}$, we immediately find

$$
x-\kappa=\sum_{i} \alpha_{i}+\gamma^{-1}(x-\alpha) \sum_{i} \gamma_{i} \cosh \varphi_{i}+\left|1-\left(\frac{x-\alpha}{\gamma}\right)^{2}\right|^{1 / 2} \sum_{i} \gamma_{i} \sinh \varphi_{i} .
$$

Therefore we reach the following compatibility conditions:

$$
\begin{aligned}
\sum_{i} \alpha_{i}+\kappa & =\alpha, \\
\sum_{i} \gamma_{i} \cosh \varphi_{i} & =\gamma, \\
\sum_{i} \gamma_{i} \sinh \varphi_{i} & =0 .
\end{aligned}
$$

Eq. (20a) gives the value of $\alpha$ in terms of $\left\{\alpha_{j}\right\}$ and Eq. (20b) consistently provides the value of the Casimir by means Eq. (13). Eq. (20c), which imposes the constraint

$$
\sum_{i} \sinh \varphi_{i}\left(\alpha_{i}^{2}+E^{-1} b_{i}\right)^{1 / 2}=0
$$

on the the parameters $\left\{\alpha_{i}, \varphi_{j}\right\}$ by virtue of Eq. (17), is responsible for the fact that only $2 n-1$ among these parameters have been included in the set (19). Note that Eqs. (12) and (20a) can be combined to express $E$ as a function of $\left\{\alpha_{i}\right\}$ as

$$
E=-\frac{c}{\kappa+2 \sum_{i} \alpha_{i}} .
$$

Finally, this permits to express $\gamma_{i}$ in terms of $\left\{\alpha_{j}\right\}$ by means of Eq. (17). We omit the discussion of the case $E \leq 0$, which goes along the same lines.

\section{The geometric content of $\mathcal{H}$}

It is apparent that $\mathcal{H}$ is a (nonconstant) multiple of the SW system. What we want to stress in this section is that their connection does not end here: $\mathcal{H}$ is in fact the intrinsic SW Hamiltonian in the manifold $\mathcal{M}^{n}$. And what makes this space remarkable is that its intrinsic SW model is also MS.

It is apparent from Eq. (3) that the Riemannian manifold $\mathcal{M}^{n}$ is spherically symmetric, this $\mathrm{SO}(n)$ symmetry being a consequence of the $\mathfrak{s l}(2)$ coalgebra structure outlined in Sec. 2. Its curvature is certainly nonconstant; for the sake of completeness we note that its scalar curvature is negative and given by

$$
R=-(n-1) \frac{3(n-2) \mathbf{q}^{2}+2 \kappa n}{\left(\kappa+\mathbf{q}^{2}\right)^{3}} .
$$


One can define intrinsic versions of the Kepler and harmonic oscillator potentials on $\mathcal{M}^{n}$. To this end, denote by $\Delta_{\mathcal{M}^{3}}$ the Laplace-Beltrami operator on $\mathcal{M}^{3}$ and let $V_{3} \in C^{\infty}\left(\mathcal{M}^{3} \backslash\{\mathbf{0}\}\right)$ be its (minimal symmetric) Green function, i.e., a function $V_{3}(\mathbf{q})=v(|\mathbf{q}|)$ satisfying

$$
-\Delta_{\mathcal{M}^{3}} V_{3}=\delta_{\mathcal{M}^{3}} .
$$

Here $v \in C^{\infty}\left(\mathbb{R}^{+} \backslash\{0\}\right)$ and $\delta_{\mathcal{M}^{3}}$ stands for the delta distribution in $\mathcal{M}^{3}$ supported at the origin. It can be proved [13] that such a function exists and is unique.

Definition 4. The Kepler and harmonic oscillator potentials in $\mathcal{M}^{n}$ are

$$
V_{\text {Kepler }}(\mathbf{q}):=K v(|\mathbf{q}|), \quad V_{\text {Harm }}(\mathbf{q}):=K v(|\mathbf{q}|)^{-2},
$$

where $K \in \mathbb{R}$ is an arbitrary constant.

Remark 5. The above definition, which is based on the potential theory of these spaces, can be easily extended to any spherically symmetric Riemannian space with the appropriate behavior at infinity [30]. Such a prescription reproduces the intrinsic Kepler and harmonic potentials on constant curvature spaces (see [12] and references therein). The case of U(2)-symmetric Kähler 4-manifolds, which had already appeared in the literature [33], is based on the same ideas but does not fit into this framework. Certainly the above prescription does not generally lead to MS potentials.

Let $\mathbf{q} \in \mathcal{M}^{3}$ and define $r=|\mathbf{q}|$. It can be readily verified that the action of the Laplace-Beltrami operator in $\mathcal{M}^{3}$ on some function $f(r)$ is

$$
\Delta_{\mathcal{M}^{3}} f=\frac{1}{r^{2}\left(\kappa+r^{2}\right)} \frac{\mathrm{d}}{\mathrm{d} r}\left(r^{2} \sqrt{\kappa+r^{2}} \frac{\mathrm{d} f}{\mathrm{~d} r}\right) .
$$

By setting the above expression equal to zero for $r>0$ it is straightforward to find that the function $v$ defined above is

$$
v(r)=\frac{\sqrt{\kappa+r^{2}}}{r}
$$

up to a multiplicative constant. Hence the Kepler and harmonic oscillator potentials in $\mathcal{M}^{n}$ are respectively given by

$$
V_{\text {Kepler }}(\mathbf{q})=K \frac{\sqrt{\kappa+\mathbf{q}^{2}}}{|\mathbf{q}|}, \quad V_{\mathrm{Harm}}(\mathbf{q})=\frac{K \mathbf{q}^{2}}{\kappa+\mathbf{q}^{2}} .
$$

This shows that the MS Hamiltonian (1) is in fact the intrinsic SW systems, i.e.,

$$
\mathcal{H}(\mathbf{p}, \mathbf{q})=\|\mathbf{p}\|_{\mathcal{M}^{n}}^{2}+V_{\text {Harm }}(\mathbf{q})+\left(\kappa+\mathbf{q}^{2}\right)^{-1} \sum_{j} b_{j} q_{j}^{-2},
$$

with $\|\cdot\|_{\mathcal{M}^{n}}$ representing the metric on the cotangent bundle of $\mathcal{M}^{n}$. 


\section{Acknowledgements}

This work was partially supported by the Spanish MEC and the Junta de Castilla y León under grants no. FIS2004-07913 and VA013C05 (A.B. and F.J.H.), by the Spanish DGI under grant no. FIS2005-00752 (A.E.) and by the INFN-CICyT (O.R.). Furthermore, A.E. acknowledges the financial support of the Spanish MEC through an FPU scholarship, as well as the hospitality and the partial support of the Physics Department of Roma Tre University.

\section{References}

[1] M. A. Agrotis, P. A. Damianou, C. Sophocleous, The Toda lattice is superintegrable, Physica A 365 (2006) 235-243.

[2] V. I. Arnold, V. V. Kozlov, A. I. Neishtadt, Mathematical aspects of classical and celestial mechanics, Springer-Verlag, Berlin, 1997.

[3] A. Ballesteros, F. J. Herranz, Universal integrals for superintegrable systems on $N$-dimensional spaces of constant curvature, J. Phys. A: Math. Theor. 40 (2007) F51-F59.

[4] A. Ballesteros, F. J. Herranz, M. Santander, T. Sanz-Gil, Maximal superintegrability on $N$-dimensional curved spaces, J. Phys. A: Math. Gen. 36 (2003) L93-L99.

[5] A. Ballesteros, F. Musso, O. Ragnisco, Classical and quantum integrable systems: the coalgebra approach, Regul. Chaotic Dyn. 4 (2002) 393-398.

[6] A. Ballesteros, O. Ragnisco, A systematic construction of completely integrable Hamiltonians from coalgebras, J. Phys. A: Math. Gen. 31 (1998) 3791-3813.

[7] J. Bertrand, Mécanique analytique, C. R. Acad. Sci. 77 (1873) 849-853.

[8] A. T. Bruce, R. G. McLenaghan, R. G. Smirnov, A geometrical approach to the problem of integrability of Hamiltonian systems by separation of variables, J. Geom. Phys. 39 (2001) 301-322.

[9] F. Calogero, Solution of the one-dimensional $N$-body problems with quadratic and/or inversely quadratic pair potentials, J. Math. Phys. 12 (1971) 419-436.

[10] F. Calogero, Two new classes of isochronous Hamiltonian systems, J. Nonlin. Math. Phys. 11 (2004) 208-222.

[11] J. F. Cariñena, M. F. Rañada, M. Santander, A nonlinear deformation of the isotonic oscillator and the Smorodinski-Winternitz system: integrability and superintegrability, Regul. Chaotic Dyn. 10 (2005) 423-436.

[12] J. F. Cariñena, M. F. Rañada, M. Santander, Central potentials on spaces of constant curvature: the Kepler problem on the two-dimensional sphere $S^{2}$ and the hyperbolic plane $H^{2}$, J. Math. Phys. 46 (2005) 52702(26). 
[13] A. Enciso, D. Peralta-Salas, Geometrical and topological aspects of electrostatics on Riemannian manifolds, submitted for publication.

[14] N. W. Evans, Superintegrability in classical mechanics, Phys. Rev. A 41 (1990) 5666-5576.

[15] N. W. Evans, Superintegrability of the winternitz system, Phys. Lett. A 147 (1990) 483-486.

[16] N. W. Evans, Group theory of the Smorodinsky-Winternitz system, J. Math. Phys. 32 (1991) 3369-3375.

[17] I. Fris, V. Mandrosov, J. Smorodinsky, M. Uhlir, P. Winternitz, On higherorder symmetries in quantum mechanics, Phys. Lett. 16 (1965) 354-356.

[18] C. Gonera, Isochronic potentials and new family of superintegrable systems, J. Phys. A: Math. Gen. 37 (2004) 4085-4095.

[19] Y. I. Granovski, I. M. Lutzenko, A. S. Zhedanov, Mutual integrability, quadratic algebras, and dynamical symmetry, Ann. Phys. 217 (1992) 1-20.

[20] S. Gravel, P. Winternitz, Superintegrability with third-order integrals in quantum and classical mechanics, J. Math. Phys. 43 (2002) 5902-5912.

[21] F. J. Herranz, A. Ballesteros, Superintegrability on three-dimensional Riemannian and relativistic spaces of constant curvature, SIGMA 2 (2006) $010(22)$.

[22] A. Huaux, Sur la séparation des variables dans l'équation aux dérivées partielles de Hamilton-Jacobi, Ann. Mat. Pura Appl. 108 (1976) 251-282.

[23] E. G. Kalnins, Separation of variables for Riemannian spaces of constant curvature, Wiley, New York, 1986.

[24] E. G. Kalnins, J. M. Kress, W. Miller, Second-order superintegrable systems in conformally flat spaces. IV. The classical 3D Stäckel transform and 3D classification theory, J. Math. Phys. 44 (2006) 43514(40).

[25] E. G. Kalnins, J. M. Kress, W. Miller, P. Winternitz, Superintegrability in a two-dimensional space of nonconstant curvature, J. Math. Phys. 43 (2002) 970-983.

[26] E. G. Kalnins, J. M. Kress, W. Miller, P. Winternitz, Superintegrable systems in Darboux spaces, J. Math. Phys. 44 (2003) 5811-5848.

[27] E. G. Kalnins, W. Miller, The theory of orthogonal $R$-separation for Helmholtz equations, Adv. Math. 51 (1984) 91-106.

[28] E. G. Kalnins, G. C. Williams, W. Miller, G. S. Pogosyan, On superintegrable symmetry-breaking potentials in $N$-dimensional Euclidean space, J. Phys. A: Math. Gen. 35 (2002) 4755-4773.

[29] G. Koenigs, Sur les géodésiques à intégrales quadratiques, in: G. Darboux (Ed.), Leçons sur la théorie générale des surfaces, Vol. 4, Chelsea, New York, 1972, pp. 368-404. 
[30] P. Li, L. F. Tam, Symmetric Green's functions on complete manifolds, Amer. J. Math. 109 (1987) 1129-1154.

[31] H. Liebmann, Nichteuklidische Geometrie, Walter de Gruyter, Berlin, 1923.

[32] J. Moser, Three integrable Hamiltonian systems connected with isospectral deformations, Adv. Math. 16 (1975) 197-220.

[33] A. Nersessian, A. Yeranyan, Three-dimensional oscillator and Coulomb systems reduced from Kähler spaces, J. Phys. A: Math. Gen. 37 (2004) 27912801.

[34] M. F. Rañada, Superintegrable $n=2$ systems, quadratic constants of motion and the potentials of Drach, J. Math. Phys. 38 (1997) 4165-4178.

[35] M. B. Sheftel, P. Tempesta, P. Winternitz, Superintegrable systems in quantum mechanics and classical Lie theory, J. Math. Phys. 42 (2001) 659 673.

[36] B. Sutherland, Exact results for a quantum many-body problem in one dimension, Phys. Rev. A 4 (1971) 2019-2021.

[37] M. Toda, One-dimensional dual transformation, J. Phys. Soc. Japan 22 (1967) 431-436.

[38] S. Wojciechowski, Superintegrability of the Calogero-Moser system, Phys. Lett. A 95 (1983) 279-281. 